\title{
Dickkopf-3 and $\beta$-catenin play opposite roles in the Wnt/ $\beta$-catenin pathway during the abnormal subchondral bone formation of human knee osteoarthritis
}

\author{
XUEGANG LIANG $^{1,2}$, QUNHUA JIN ${ }^{1}$, XIAOCHUN YANG ${ }^{1}$ and WENHUI JIANG ${ }^{3}$ \\ ${ }^{1}$ Department of Orthopedics, Ningxia Medical University General Hospital; ${ }^{2}$ Department of Orthopedics, \\ People's Hospital of Ningxia Hui Autonomous Region Affiliated to Ningxia Medical University, \\ Yinchuan, Ningxia Hui Autonomous Region 750000; ${ }^{3}$ Clinical Medical College, \\ Xi'an Medical College, Xi'an, Shanxi 710000, P.R. China
}

Received November 30, 2021; Accepted January 28, 2022

DOI: $10.3892 /$ ijmm.2022.5103

\begin{abstract}
Osteoarthritis (OA) is condition which poses a main concern to the aging population and its severity is expected to increase with the increasing life expectancy. In the future, several possible targets for OA treatment need to be defined. Dickkopf-related protein 3 (DKK3) is an atypical member of the Wnt-antagonistic dickkopf-related protein (DKK) family. The availability of research into the role of DKK3 in the abnormal remodeling of subchondral bone in human knee joints is currently limited. Thus, the aim of the present study was the evaluation of DKK3 expression in the abnormal bone remodeling of subchondral bone in human knee OA in order to clarify the role of DKK3 in subchondral bone remodeling and to acknowledge its potential relevance to $\beta$-catenin. In total, 38 specimens were collected from osteotomies of the medial tibial plateau of the human knee. The patient samples were then divided into the normal, mild, moderate and severe symptom groups, according to the Osteoarthritis Research Society International (OARSI) score. Following hematoxylin and eosin (H\&E) and Safranin O-fast green staining for alkaline phosphatase (AZO method), changes in the distribution and number of osteocytes in the subchondral bone and the degree of sclerosis of the subchondral bone were observed. Immunohistochemical staining, immunofluorescence, western blot analysis and reverse-transcription quantitative PCR (RT-qPCR) were used for the detection of DKK3 and $\beta$-catenin expression level changes in osteoblasts in the subchondral bone of the medial tibial plateau. H\&E and alkaline phosphatase staining revealed that the total number of osteocytes in the
\end{abstract}

Correspondence to: Dr Qunhua Jin, Department of Orthopedics, Ningxia Medical University General Hospital, 804 Shengli South Street, Xingqing, Yinchuan, Ningxia Hui Autonomous Region 750000, P.R. China

E-mail: 407323266@qq.com

Key words: osteoarthritis, Dickkopf-3, $\beta$-catenin, Wnt/ $\beta$-catenin signaling, osteoblasts, sclerosis, subchondral bone subchondral bone increased with the severity of the disease. The samples were also evaluated using Safranin O-Fast Green staining and were attributed a score according to the OARSI scoring system: The scoring number and cartilage damage increased along with OA severity. Immunohistochemistry and immunofluorescence assays demonstrated that $\beta$-catenin expression in osteocytes increased from mild to moderate, whereas DKK3 expression decreased with the development of arthritis from normal, mild to moderate. According to the results of western blot analysis, $\beta$-catenin expression was higher in moderate OA and then decreased in severe OA. On the other hand, the DKK3 levels decreased along with the progression from normal, mild to moderate OA. The results of RT-qPCR demonstrated that $\beta$-catenin and DKK3 gene expression differed with the degree of OA. On the whole, the present study demonstrates that DKK 3 and $\beta$-catenin may play opposite roles in OA subchondral bone remodeling.

\section{Introduction}

The most common orthopedic diseases include osteoarthritis (OA) and osteoporosis. OA is defined as a joint disease, characterized by cartilage degeneration, abnormal remodeling of subchondral bone and joint pain (1), and occurs in aging populations. However, previous research has demonstrated that subchondral bone hardens and cartilage degenerates during disease proression. Anderson-MacKenzie et al (2) reported that the role of subchondral bone was mainly to reduce impact forces and mitigate cartilage damage. In addition, subchondral bone density and cartilage thickness are positively associated in knees without OA, and negatively associated in knees with $\mathrm{OA}$, in support of the subchondral bone/chondral functional unit theory. These conclusions also contribute to the improvement of the understanding of the pathophysiology of OA (3). Furthermore, Neve et al (4) studied osteoblasts, which are mononuclear mesenchymal stem cell-derived cells responsible for osteogenesis and mineralization in early bone formation and subsequent bone remodeling, while OA osteoblasts exhibit an abnormal metabolic behavior. Maruotti et al (5) revealed that osteoblast disorders play a crucial role in the pathogenesis 
of OA, stimulating the osteoblast-induced production of large transcription factor quantities, growth factors and other proteins involved in OA pathogenesis. In addition, Di Pompo et al (6) previously reported that osteoblasts promoted the secretion of osteoclastic cytokines and inflammatory mediators in an acidic environment ( $\mathrm{pH}$ 6.8), further exacerbating OA.

The signaling pathways form a complex network of molecular interactions in osteoblasts. It has been reported that the Wnt signaling pathway is involved in OA, in which signaling molecules and regulatory factors are aberrantly activated or inhibited (7). The activation of the classical $\mathrm{Wnt} / \beta$-catenin signaling pathway increases subchondral bone remodeling and osteophyte formation, which are two pathological processes in bone also associated with OA progression (8). Similarly, decreased levels of Wnt activity are important for maintaining the viability of osteocytes (both chondrocytes and osteoblasts); however, excessive activation has been found to be associated with joint damage (9).

Osteoblasts and their precursors can control various Wnt ligands and are involved in the regulation of bone remodeling; the Wnt signaling may regulate chondrocyte and osteoblast differentiation and function via classical ( $\beta$-catenin-dependent) or non-classical Wnt signaling pathways, as well as cross-linking with other signaling pathways, affecting cartilage and bone metabolism. In addition, the excessive activation of the Wnt signaling pathway may exacerbate cartilage OA-like degeneration $(10,11) . \beta$-catenin has been reported to be essential for the differentiation of osteoblasts, and the overexpression of constitutively active $\beta$-catenin forms may also lead to the development of OA, characterized by increased chondrocyte hypertrophy $(12,13)$. Thus, the dysregulation of the Wnt/ $\beta$-catenin signaling pathway may lead to OA.

The Dickkopf (DKK) family has been reported to regulate the Wnt signaling pathway by binding to the Wnt receptor complex (14). Chen et al (15) demonstrated that the local injection of DKK1 knockout lentivirus into the rat femur alleviated the progression of AONFH; Wnt/ $\beta$-catenin signalling was activated, the number of empty fat cavities was reduced, the blood supply was restored and bone formation was significantly increased. Among DKK family members, Dickkopf-related protein 3 (DKK3) is an atypical member of the DKK family of Wnt antagonists; human DKK-1, -2 and -4 are located in the same homology group, whereas DKK3 does not belong to this group (16). Furthermore, the mechanisms of action of DKK3 differ from those of other proteins in the DDK gene family. Lee et al (17) demonstrated that DKK3 attenuated $\beta$-catenin protein expression and its transcriptional activity via the interaction with $\beta$-transducin repeat-containing protein $(\beta-\operatorname{TrCP})$ and prevented the translocation of $\beta$-catenin to the nucleus. DKK3 expression levels in the subchondral bone, as well as its role in osteocyte formation have not yet been fully elucidated. Therefore, the aim of the present study was to investigate the DKK3 expression levels in osteoblasts in subchondral bone from human knees with OA, in order to clarify the role of DKK3 in abnormal bone remodeling in subchondral bone and to determine its potential association with $\beta$-catenin expression level changes.

\section{Materials and methods}

Materials. A total of 38 osteotomy specimens (Table I) were collected from the medial tibial plateau of the knee joint of patients from January, 2019 to October, 2020 at the Third Department of Orthopedics, General Hospital of Ningxia Medical University (Yinchuan, China). Tissue samples from three cases with a normal and five cases with a mild OA symptomatology were obtained from young patients who underwent lower limb amputations. Tissue samples from 10 cases of moderate OA symptomatology were collected from patients diagnosed with $\mathrm{OA}$ and who underwent knee arthroplasty, including single condyle replacement. Tissue samples from 20 cases with severe OA symptomatology were also collected, including a case of tibial plateau osteotomy that was diagnosed with $\mathrm{OA}$ in a patient undergoing total knee replacement. Samples were acquired from 16 male, and 22 female patients, aged 20-80 years, with an average age of 62 years. The patients included in the present study had no family history of malignant tumors, hemiplegia, or death from stroke, and no patients had undergone surgery on the ipsilateral hip. In total, 23 patients were diagnosed with hypertension (20 patients had grade I and 3 had grade II hypertension), and none were diagnosed with type 2 diabetes. In addition, 1 patient had a giant cell tumor of the ipsilateral pelvis (Jaffe grade II). During tissue collection, all samples were immediately stored in liquid nitrogen, at $-147^{\circ} \mathrm{C}$. The sample cohort was divided into two equal parts, following sample collection. The samples in the first group were fixed with neutral formalin for $24 \mathrm{~h}$, decalcified with fast decalcification liquid for 1 week, and then embedded in paraffin, as described below. Subchondral bone was retained after cartilage removal in the other sample group, and stored in a refrigerator at $-80^{\circ} \mathrm{C}$ for later use. The present study was approved by the Ethics Committee of the General Hospital of Ningxia Medical University (approval no. KYLL-2020-20; approval date, January 14, 2021), and written informed consent was obtained from all the enrolled patients.

Experimental methods. The samples were divided into the normal, mild, moderate and severe symptom groups, according to the Osteoarthritis Research Society International (OARSI) (18), and the distribution and sclerosis of medial osteoblasts were observed following hematoxylin and eosin (H\&E), Safranin O/fast green staining and alkaline phosphatase (ALP; AZO method) as described below. Along with the increase in the osteoarthritis pathology degree, alterations in the number of subchondral osteoblasts were also observed. Changes in DKK3 and $\beta$-catenin expression in the subchondral osteoblasts of the medial tibial plateau were observed and detected using immunohistochemical staining, immunofluorescence, western blot analysis and reverse transcription-quantitative PCR (RT-qPCR) as described below.

Main reagents. Anti-DKK3 antibody (EPR15611; ab186409; rabbit monoclonal) was purchased from Abcam, and $\beta$-catenin antibody (ab32572; rabbit monoclonal) and GAPDH antibody (ab9485) were obtained from Abcam. RIPA lysate, 50X cocktail, PMSF (100 mM), phosphorylated protease inhibitor, BCA protein quantitative detection kit, $5 \mathrm{X}$ protein loading buffer and the SDS-PAGE gel preparation kit were acquired from Wuhan Google Biotechnology Co., Ltd.; the protein marker was acquired from Thermo Fisher Scientific, Inc. The PVDF membrane was acquired from MilliporeSigma; and TRIzol 
Table I. Clinicopathological data of the patients in the present study.

\begin{tabular}{|c|c|c|c|c|}
\hline Patient no. & Sex & Age (years) & Severity of symptoms & Left or right knee \\
\hline 1 & M & 65 & Moderate & Right \\
\hline 2 & F & 62 & Severe & Right \\
\hline 3 & $\mathrm{M}$ & 59 & Severe & Right \\
\hline 4 & F & 40 & Normal & Left \\
\hline 5 & M & 68 & Severe & Left \\
\hline 6 & F & 41 & Mild & Left \\
\hline 7 & $\mathrm{M}$ & 80 & Severe & Right \\
\hline 8 & F & 72 & Moderate & Right \\
\hline 9 & $\mathrm{M}$ & 32 & Normal & Right \\
\hline 10 & $\mathrm{~F}$ & 66 & Severe & Right \\
\hline 11 & $\mathrm{~F}$ & 80 & Moderate & Left \\
\hline 12 & $\mathrm{M}$ & 80 & Severe & Left \\
\hline 13 & F & 78 & Severe & Right \\
\hline 14 & F & 59 & Moderate & Left \\
\hline 15 & M & 55 & Moderate & Right \\
\hline 16 & $\mathrm{M}$ & 63 & Severe & Left \\
\hline 17 & F & 61 & Moderate & Right \\
\hline 18 & $\mathrm{M}$ & 69 & Severe & Right \\
\hline 19 & F & 44 & Mild & Left \\
\hline 20 & M & 64 & Moderate & Left \\
\hline 21 & M & 72 & Moderate & Right \\
\hline 22 & $\mathrm{M}$ & 78 & Severe & Right \\
\hline 23 & $\mathrm{~F}$ & 67 & Severe & Left \\
\hline 24 & $\mathrm{M}$ & 20 & Normal & Left \\
\hline 25 & $\mathrm{M}$ & 62 & Severe & Left \\
\hline 26 & F & 67 & Severe & Right \\
\hline 27 & F & 62 & Moderate & Right \\
\hline 28 & $\mathrm{M}$ & 78 & Severe & Left \\
\hline 29 & F & 58 & Severe & Left \\
\hline 30 & $\mathrm{M}$ & 70 & Severe & Left \\
\hline 31 & $\mathrm{~F}$ & 43 & Mild & Right \\
\hline 32 & $\mathrm{M}$ & 64 & Moderate & Left \\
\hline 33 & $\mathrm{~F}$ & 68 & Severe & Left \\
\hline 34 & M & 45 & Mild & Right \\
\hline 35 & $\mathrm{M}$ & 70 & Severe & Right \\
\hline 36 & M & 79 & Severe & Left \\
\hline 37 & $\mathrm{M}$ & 76 & Severe & Right \\
\hline 38 & $\mathrm{M}$ & 40 & Mild & Right \\
\hline
\end{tabular}

Universal and Trizol ${ }^{\circledR}$ Reagent was purchased from Invitrogen; Thermo Fisher Scientific, Inc.

Histological analysis. All obtained tissues were rinsed with normal saline three times for $15 \mathrm{~min}$ each and fixed in $4 \%$ neutral formaldehyde solution for $24 \mathrm{~h}$. Subsequently, the tissues were incubated in a rapid decalcification solution (Beijing Zhuoding Biological Company) for decalcification for 7 days and immersed in PBS solution for $6 \mathrm{~h}$, with PBS replacement every $1 \mathrm{~h}$. Tissues were trimmed to a size of $\sim 5 \times 5 \times 3 \mathrm{~mm}$ and placed in a plastic embedding box for subsequent processing. The tissues were sequentially placed in 50 ,
$70,80,95$ and $100 \%$ ethanol for 10 min each at first, and subsequently in a $100 \%$ ethanol/xylene (2:1) solution, $100 \%$ ethanol/xylene (1:1) solution, $100 \%$ ethanol/xylene (1:2) solution, xylene I, II, and III for 10 min per step. The paraffin wax was melted at $60^{\circ} \mathrm{C}$ in an electric heating constant temperature incubator (constant temperature chamber; Xiaogan Yaguang Medical-electronic Technology Co., Ltd.), placed afterwards on tissue paraffin glass for embedding, and xylene was then used to melt the paraffin wax in order to create a mixed solution. The tissues were then placed consecutively in xylene/paraffin (2:1) solution, xylene/paraffin (1:1) solution, and xylene/paraffin (1:2) solution for 30 min per step. Finally, 
the tissues were placed in paraffin I and II in a thermostat at $60^{\circ} \mathrm{C}$ for $1.5 \mathrm{~h}$, respectively.

$H \& E$ staining. Tissue samples were dewaxed by soaking the sections in xylene I and II for $10 \mathrm{~min}$, rinsing with distilled water for $30 \mathrm{sec}$, then soaking consecutively in 100 (I, II), 90, 80 and $70 \%$ alcohol for $5 \mathrm{~min}$, and finally rinsing with tap water for $5 \mathrm{~min}$ three times. The samples were stained with hematoxylin (Beijing Solarbio Science \& Technology Co., Ltd.) for $5 \mathrm{~min}$ at room temperature and rinsed with running water. Samples were differentiated with $5 \%$ acetic acid for $1 \mathrm{~min}$, washed with running water for $10 \mathrm{~min}$, stained with eosin (Beijing Solarbio Science \& Technology Co., Ltd.) for $1 \mathrm{~min}$ at room temperature, and rinsed three times with running water, for $5 \mathrm{~min}$. The slices were dehydrated by placing them in 70 , 80,90 and $100 \%$ alcohol and xylene for $1 \mathrm{~min}$ each, and were then sealed with neutral glue, and covered with a cover slip. The tissues were examined using a Leica microscope (Leica DM2700 M; Leica Microsystems GmbH).

Safranin O/fast green staining. The dewaxing procedure was performed according to the H\&E staining protocol, as described above. The sections were stained with hematoxylin for $5 \mathrm{~min}$ and rinsed with tap water for $10 \mathrm{~min}$. The sections were dyed with $0.3 \%$ solid green for $3 \mathrm{~min}$ and then washed with tap water for $5 \mathrm{~min}$ three times. The slices were placed in $1 \%$ acetic acid for $5 \mathrm{sec}$, then immersed in $1 \%$ safranin staining solution (Beijing Solarbio Science \& Technology Co., Ltd.) for 2 min at room temperature, and then rinsed three times with distilled water for $5 \mathrm{~min}$. Finally, the samples were dehydrated and sealed as process as described above for H\&E staining. The samples were examined using a Leica microscope (Leica Microsystems $\mathrm{GmbH}$ ).

The final score was based on the Safranin O/fast green staining results and the OARSI scoring items. The OARIS score was obtained by multiplying the histological grading of cartilage degeneration (in 6 grades) by the histological staging of cartilage degeneration (in five stages) following Safranin $\mathrm{O} /$ fast green staining.

ALP staining ( $A Z O$ coupling method). The dewaxing procedure was performed as described above for the H\&E staining protocol. Firstly, the working fluid was prepared. ALP staining solution B1 (AS-BI staining solution) and alkaline phosphatase staining solution B2 (FBB staining solution) (both stains from Beijing Solarbio Science \& Technology Co., Ltd.) were mixed in a volume ratio of $1: 1$, and the prepared ALP incubation solution was then dropped onto the cut sections. The sections were then incubated in a dark incubator for $20 \mathrm{~min}$ at $37^{\circ} \mathrm{C}$ and washed with water for $5 \mathrm{~min}$. The sections were immersed in nuclear hard red staining solution for counterstaining for $3 \mathrm{~min}$ at room temperature. Finally, samples were dehydrated and sealed as described above previously described. The samples were examined using a Leica microscope (Leica Microsystems $\mathrm{GmbH}$ ).

Immunohistochemical staining. The dewaxing procedure was performed as described above. A total of $20 \mu 10.1 \%$ trypsin solution was added to the tissue, incubated in an incubator at $37^{\circ} \mathrm{C}$ for $15 \mathrm{~min}$, and soaked in PBS for $15 \mathrm{~min}$. Endogenous peroxidase blocker (Beijing Zhongshan Jinqiao Biological Co., Ltd.) was then added $(20 \mu 1)$ to the tissue, incubated at $37^{\circ} \mathrm{C}$ for $10 \mathrm{~min}$, and soaked in PBS solution for $15 \mathrm{~min}$, followed by the addition of goat serum (Beijing Solarbio Science \& Technology Co., Ltd.) and incubation at $37^{\circ} \mathrm{C}$ for $30 \mathrm{~min}$. Afterwards, the primary antibodies [DKK3 antibody (goat anti-rabbit, 1:100, ab186409, Abcam) and $\beta$-catenin antibody (goat anti-rabbit, 1:150, ab32572, Abcam)] were added in a dropwise manner to the tissue and incubated overnight in a refrigerator at $4{ }^{\circ} \mathrm{C}$. The secondary antibody used was enhanced goat anti-rabbit IgG polymer (1:500, PV-9000, $37^{\circ} \mathrm{C}, 40 \mathrm{~min}$; Beijing Zhongshan Jinqiao Biological Co., Ltd.). The slides were removed warmed to $37^{\circ} \mathrm{C}$ for $40 \mathrm{~min}$ the following day. Immunohistochemical detection reagent 2 (Beijing Zhongshan Jinqiao Biological Co., Ltd.) was then added in a dropwise manner to the tissues. The tissues were incubated in an incubator at $37^{\circ} \mathrm{C}$ for $30 \mathrm{~min}$ and soaked in PBS solution for $15 \mathrm{~min}$. Immunohistochemical detection reagent 3 (Beijing Zhongshan Jinqiao Biological Co., Ltd.) was dropped onto the tissues. The samples were placed in an incubator at $37^{\circ} \mathrm{C}$ and incubated for $40 \mathrm{~min}$. Subsequently, the samples were removed from the incubator and soaked in PBS solution for $15 \mathrm{~min}$ at room temperature. DAB chromogenic solution (Beijing Zhongshan Jinqiao Biological Co., Ltd.) was then added in a dropwise manner to the tissues, and they were observed under an optical microscope (Leica Microsystems $\mathrm{GmbH}$ ) for $1 \mathrm{~min}$, and soaked in distilled water for $5 \mathrm{~min}$. The cells were then stained with hematoxylin for $5 \mathrm{~min}$ at room temperture, differentiated with $5 \%$ acetic acid for $1 \mathrm{~min}$, and rinsed with tap water for $10 \mathrm{~min}$. Samples were dehydrated and sealed as described in the H\&E staining section above. Finally, analysis was performed based on the experimental results. Tissues were counted in areas of high expression, and with the use of ImageJ (1.8.0) software (National Institutes of Health) was used to determine the intensity of protein expression. Afterwards, the histological score was calculated as the average optical density of positively stained osteoblasts, which equals to the ratio of the overall optical density of the positive cells to the positive area. Positive cells are those with clear brownish-yellow granules in the cytoplasm or nucleus.

Immunofluorescence. The procedure for the first day of the immunofluorescence protocol was the same as that used for immunohistochemistry (described above). The following day, the slices were warmed to room temperature for $40 \mathrm{~min}$. Under dark conditions, goat anti-rabbit IgG H\&L (Cy3; S0011, Affinity Biosciences, Ltd.) fluorescent secondary antibody (1:500) was added to the tissues and incubated for $1 \mathrm{~h}$ at $37^{\circ} \mathrm{C}$. The samples were then soaked and washed in PBS for $15 \mathrm{~min}$. DAPI (Beijing Solarbio Science \& Technology Co., Ltd.) was then added in a dropwise manner in the dark and incubated at room temperature for $5 \mathrm{~min}$ at room temperature. The samples were then soaked in PBS solution for $4 \mathrm{~min}$, followed by anti-fluorescence attenuation sealer (containing DAPI) drops on the tissue for mounting. The samples were examined using a Leica microscope (DM4B; Leica Microsystems GmbH). For immunofluorescence analysis, in a place with high light intensity, under a 40X lens, the optical density value was analyzed under the same area and was equal to the ratio of the overall optical density to the area. 
A
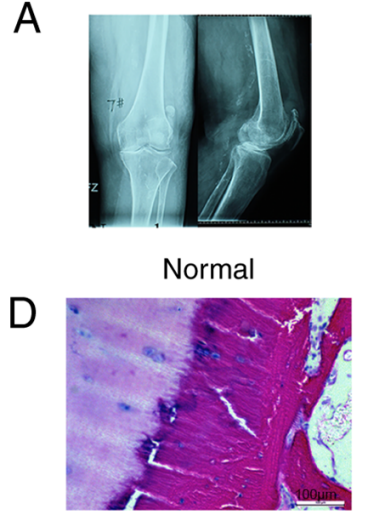

F

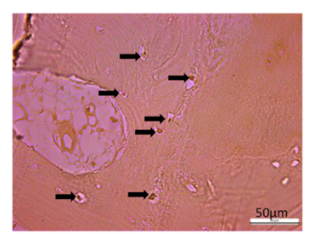

B

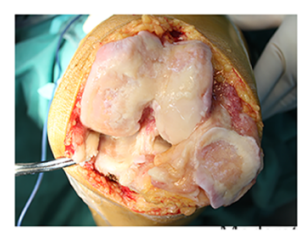
Mild
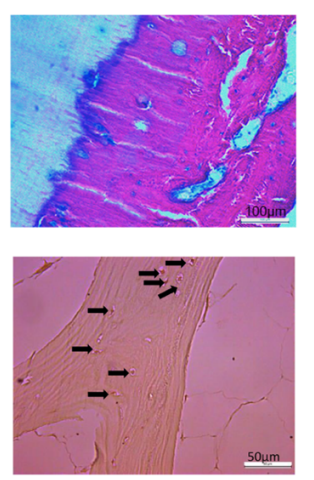

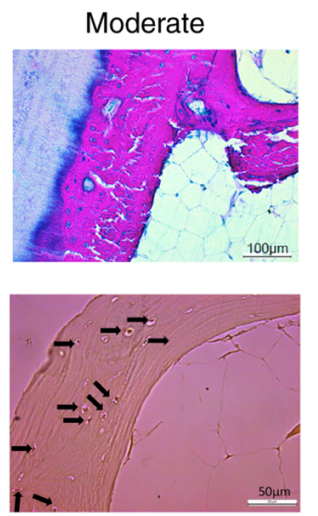

C

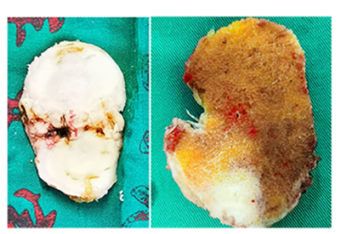

Severe
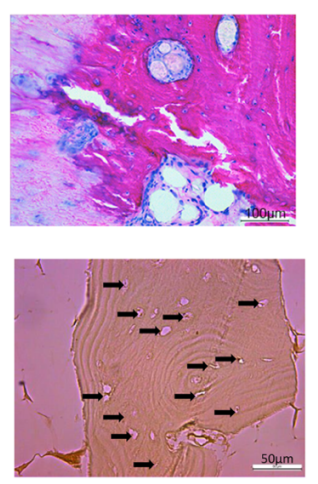

E

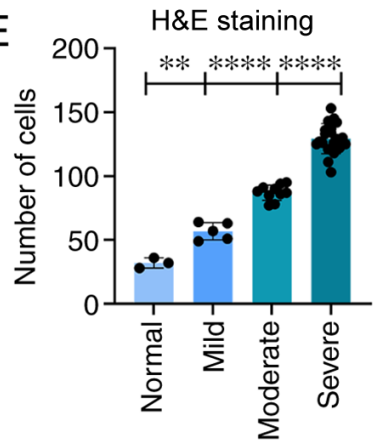

G

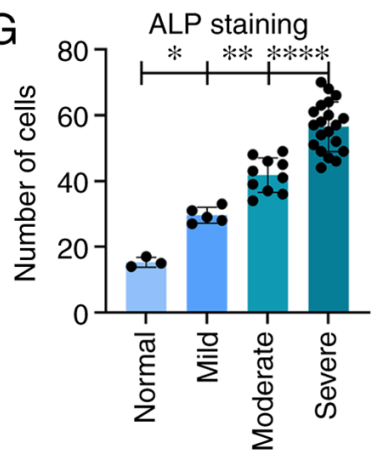

Figure 1. (A) Preoperative radiographs of the patient. (B) Intraoperative gross pathological images. (C) Intraoperative intercepted bone plates of the tibial plateau including cartilage. (D) Subchondral bone H\&E staining. (F) Subchondral bone ALP staining of the subchondral bone tissue. The arrows indicate cells positive for ALP staining. (E and G) Results of cell number statistical analysis. In H\&E staining and ALP staining, there was a statistically significant difference between the groups $\left({ }^{*} \mathrm{P}<0.05,{ }^{* * * * *} \mathrm{P}<0.0001\right.$, and $\left.{ }^{* * *} \mathrm{P}<0.01\right)$. (E and $\left.\mathrm{G}\right)$ The data were analyzed using one-way ANOVA; mean of the $95 \%$ confidence interval: Normal $(n=3)$, mild $(n=5)$, moderate $(n=10)$ and severe $(n=20)$. ALP, alkaline phosphatase; H\&E, hematoxylin and eosin.

Western blot analysis. All tissues used were removed from $-80^{\circ} \mathrm{C}$. Subsequently, the samples were washed with cold PBS 2-3 times for blood stain removal, cut into small sections $(3 \times 3 \times 5 \mathrm{~mm})$ using a tissue grinder, and placed in a homogenization tube (stored in a liquid nitrogen tank). Subsequently, a total protein extraction kit [containing lysate, phosphatase inhibitor (100X), protease inhibitor (100X), PMSF (100X; Beijing Zhongshan Jinqiao Biological Co.)] was applied for protein extraction. The homogenization tube was placed in the homogenizer, the program was selected to homogenize thoroughly so that the bone texture was $120 \mathrm{sec}$ and was intermittently cooled during the period (liquid nitrogen was automatically added intermittently). The homogenized sample tube was removed, iced for $30 \mathrm{~min}$, and shaken every $5 \mathrm{~min}$ to ensure that the tissue was completely lysed. The supernatant, which is the total protein solution, was collected and centrifuged at $4^{\circ} \mathrm{C}$ at $16,000 \mathrm{x}$ g for $10 \mathrm{~min}$. The undenatured protein solution was determined using the BCA protein concentration determination kit method and the microplate reader [ESCO (Shanghai) Enterprise Development Co., Ltd.] was used to measure the protein concentration (referring to the kit instructions for the specific method), and the protein concentration was then calculated. The protein solution was added to $5 \mathrm{X}$ protein loading buffer at a ratio of 4:1 and denatured in a boiling water bath for $15 \mathrm{~min}$. Subsequently, a $10 \%$ SDS-PAGE gel was prepared and $15 \mu 1$ protein per sample was loaded in equal volumes of sample (including markers) into the gel wells. Electrophoresis was performed $(80 \mathrm{~V}$ for concentrated gel, $120 \mathrm{~V}$ for separation gel, $300 \mathrm{~mA}$ for $40 \mathrm{~min}$ ) and gel was transferred to a PVDF membrane (MilliporeSigma). The membrane was washed with TBST three times for $5 \mathrm{~min}$, blocked with $5 \%$ skim milk for $1.5 \mathrm{~h}$ at room temperature, and incubated with the primary antibodies [anti-DKK3 antibody (ab186409; rabbit monoclonal, 1:300), anti-GAPDH antibody (ab9485, Abcam, rabbit monoclonal, 1:300) and anti- $\beta$-catenin antibody (ab32572, Abcam, rabbit monoclonal, 1:200)] at $4^{\circ} \mathrm{C}$ overnight. On the second day, the samples were moved to room temperature conditions for $40 \mathrm{~min}$ and the membrane was then washed with TBST three times for $5 \mathrm{~min}$, incubated with the secondary antibody [anti-horseradish peroxidase antibody $(1: 1,000$, ab181658, Abcam)] at room temperature for $1.5 \mathrm{~h}$. Afterwards, the membranes were washed with TBST three times for $5 \mathrm{~min}$. Finally, ECL luminescent agent (Beijing Solarbio Science $\&$ Technology Co., Ltd.) was used for exposure and observation in a chemical exposure machine. Densitometry was performed using Image J (1.8.0) software (National Institutes of Health). Western blot analysis utilized statistical analysis based on the gray value obtained, which is equal to the ratio of the gray value of the target protein to the gray value of the internal reference.

Reverse transcription-quantitative polymerase chain reaction ( $R T-q P C R$ ). Total RNA was isolated from tissues using a high-speed homogenizer for cancellous bone tissue comminution, using TRIzol ${ }^{\circledR}$ reagent (Invitrogen; Thermo Fisher Scientific, Inc.). RNA (1 $\mu \mathrm{g})$ was then reverse transcribed into cDNA using the PrimeScript RT kit (Affinity Biosciences, Ltd.) according to the manufacturer's protocol. The cDNA samples were then analyzed using LightCycler 480 SYBR-Green I Master (Roche Diagnostics) for RT-qPCR on a LightCycler 480 system (Roche Diagnostics). The reaction 

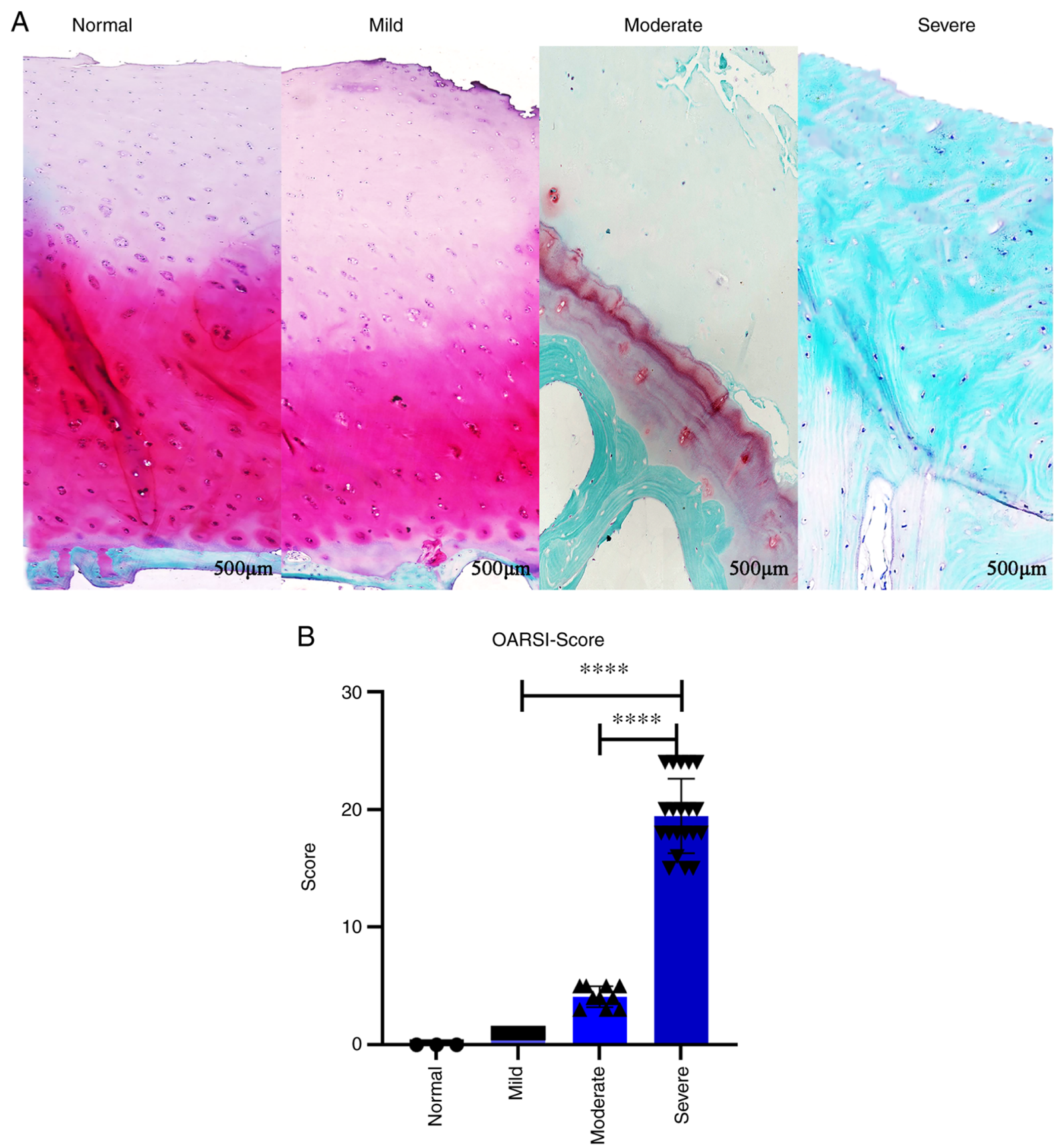

Figure 2. (A) Safranin O staining: Each image demonstrates the OARSI histological score of the medial tibial plateau cartilage. (B) Statistical analysis results. The difference between the mild and moderate groups was statistically significant $\left({ }^{* * * *} \mathrm{P}<0.0001\right)$. (B) Data were analyzed using the Kruskal-Wallis test. OARSI score equals cartilage degenerative tissue grading times staging. Normal $(n=3)$, mild $(n=5)$, moderate $(n=10)$ and severe $(n=20)$. OARSI, Osteoarthritis Research Society International.

conditions were as follows: $95^{\circ} \mathrm{C}$ for $30 \mathrm{sec}$, followed by $5 \mathrm{sec}$ at $95^{\circ} \mathrm{C}, 20 \mathrm{sec}$ at $60^{\circ} \mathrm{C}$ and $20 \mathrm{sec}$ at $75^{\circ} \mathrm{C} 40$ cycles, and GAPDH expression was used as an internal control. The data was analyzed for expression changes using the $2^{-\Delta \Delta C q}$ formula (19). The primer sequences for $\beta$-catenin, DKK3 and GAPDH were as follows: $\beta$-catenin forward, 5'-CAT CTACACAGTTTGATGCTGCT-3' and reverse, 5'-GCA GTTTTGTCAGTTCAGGGA-3'; DKK3 forward, 5'-CAA TGGGACCATCTGTGACAAC-3' and reverse, 5'-ATCGGT CCAAGGCTCCATCA-3'; GAPDH forward, 5'-TGCCAA ATGATGACATCAAGAA-3' and reverse, 5'-GGAGTGGGT GTCGCTGTTG-3'.
Statistical analysis. All data were analyzed using SPSS 22.0 (IBM Corp.) statistical software. Graphs were drawn using GraphPad Prism software (version 8.0). ImageJ (1.8.0) (National Institutes of Health) software was used for immunohistochemistry, immunofluorescence, and western blot analysis. All data were tested for normality of distribution using the Shapiro-Wilk test. One-way analysis of variance with Tukey's post hoc test was used to compare the parametric data between groups. For non-parametric data, the Kruskal-Wallis test with Dunn's post hoc test were used. A value of $\mathrm{P}<0.05$ was considered to indicate a statistically significant difference. Data from all studies were subjected to before statistical analysis. 
A
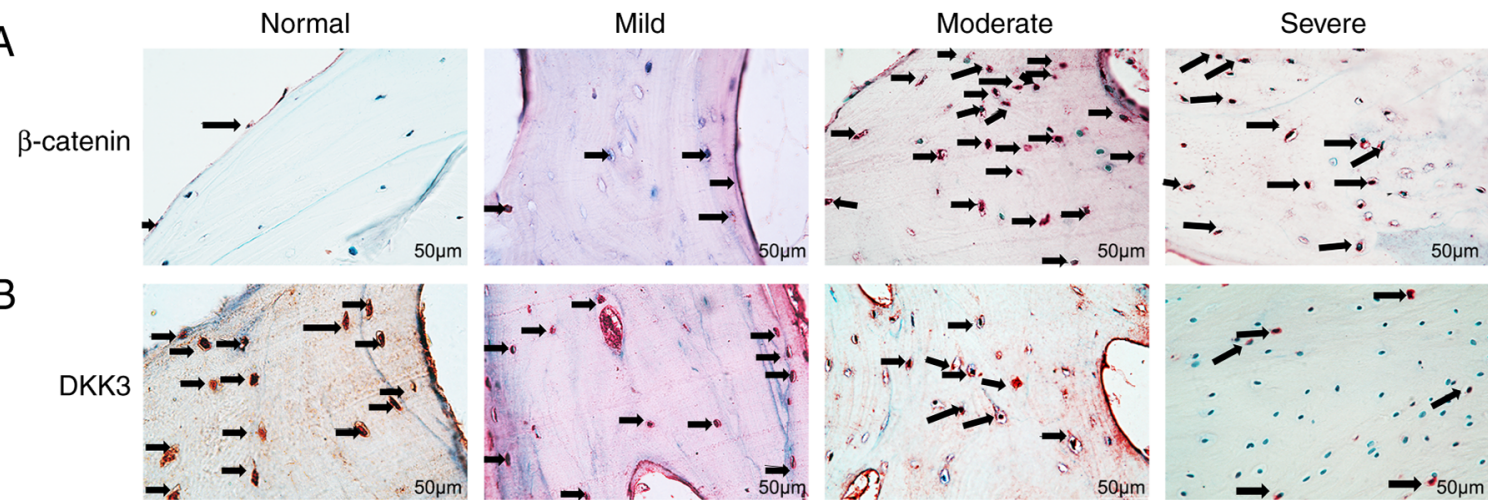

C
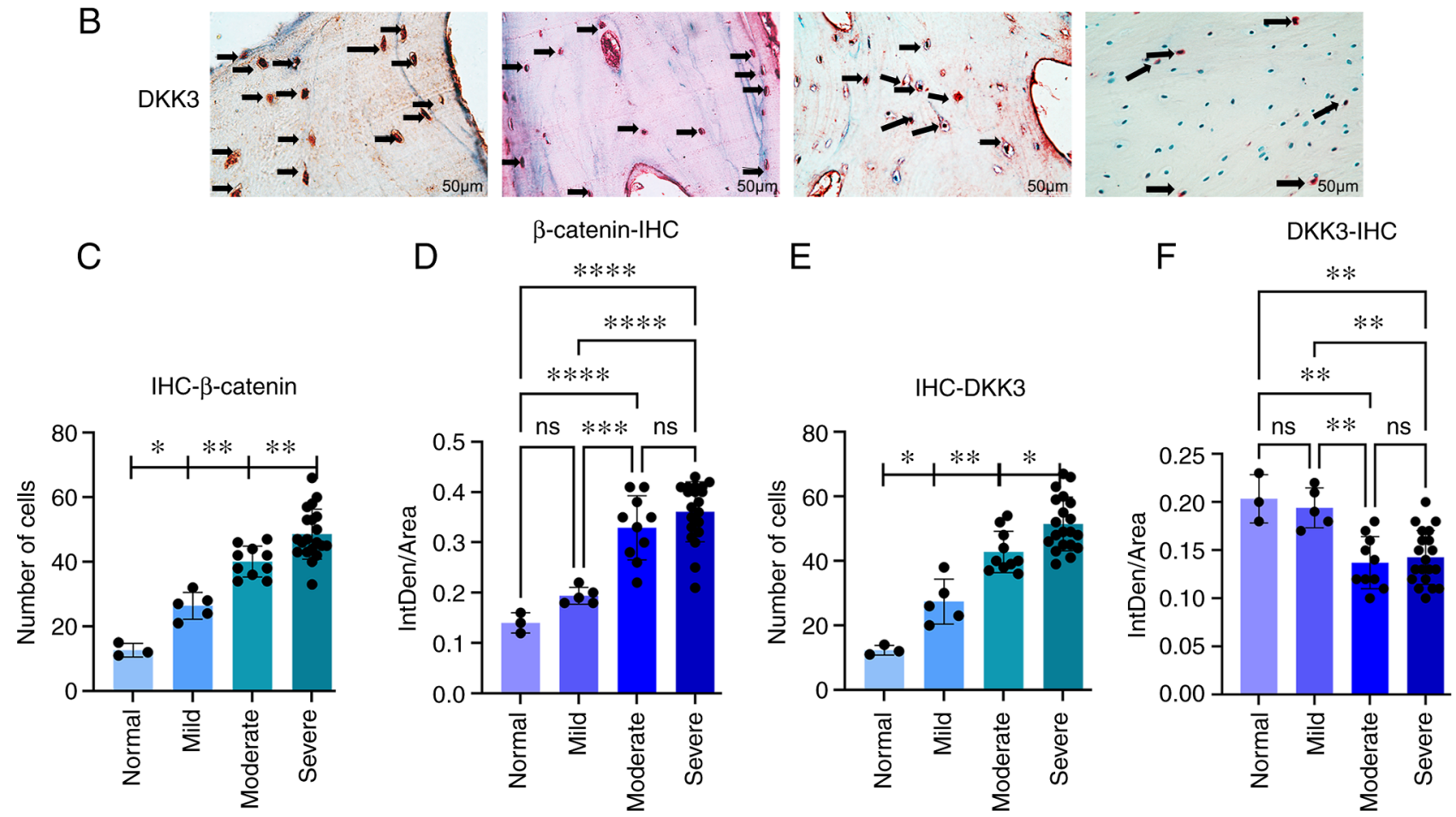

Figure 3. (A and B) Immunohistochemical staining. Arrows indicate the number of positively stained cells. (C) In the $\beta$-catenin group, the difference in cell number was statistically significant between the normal and mild, mild and moderate, and moderate and severe symptom groups $\left({ }^{*} \mathrm{P}<0.01,{ }^{* *} \mathrm{P}<0.01\right.$ and ${ }^{* *} \mathrm{P}<0.01$ respectively). (D) Statistically significant differences between the mild and moderate symptom groups according to the average optical density values $\left({ }^{* * *} \mathrm{P}<0.001\right.$ and $\left.^{* * * * *} \mathrm{P}<0.0001\right)$. (E) In the DKK3 group, cell numbers were statistically significant between the normal and mild, mild and moderate, and moderate and severe symptom groups $\left({ }^{*} \mathrm{P}<0.05,{ }^{* *} \mathrm{P}<0.01\right.$ and ${ }^{*} \mathrm{P}<0.05$, respectively). (F) Statistically significant differences between the mild and moderate groups according to the average optical density values $\left({ }^{* *} \mathrm{P}<0.01\right)$. (C-F) Data were analyzed using one-way ANOVA; mean of the $95 \%$ confidence interval: Normal $(n=3)$, mild $(n=5)$, moderate $(n=10)$ and severe $(n=20)$. DKK3, Dickkopf-related protein 3; ns, not significant.

\section{Results}

Cell count and changes in osteoblast activation. Histological samples of the medial tibial plateau were collected and divided into the normal $(n=3)$, mild $(n=5)$, moderate $(n=10)$ and severe symptom groups $(n=20)$. From the analysis of the pre-operative radiographs from the patients (Fig. 1A), gross pathological images (Fig. 1B) and intraoperative cuttings of the bone plate of the tibial plateau, including cartilage and subchondral bone (Fig. 1C), the medial subchondral bone was found to be markedly sclerotic and cartilage damage was evident. To detect changes in the number of bone cells in the subchondral bone, $\mathrm{H} \& \mathrm{E}$ staining was performed. The results revealed changes in the H\&E staining of the subchondral bone tissue (Fig. 1D), and combined with statistical analysis (Fig. 1E) there was a statistically significant difference in cell numbers between the normal, mild and moderate/severe symptom groups. The total number of bone cells (including osteoblasts and osteoclasts) increased with the progression of OA. To observe the changes in the number of osteoblasts in the subchondral bone tissue, ALP staining of the subchondral bone tissue was performed (Fig. 1F). By observing the results of ALP staining and combining these with statistical analysis (Fig. 1G), an increase in the number of osteoblasts with calcaneal nodules in the subchondral bone was observed, in parallel with the aggravation of the disease. There was a statistically significant difference between the mild and moderate symptom groups and between the moderate and severe symptom groups. The arrows in Fig. 1F indicate the calcareous nodules within the osteoblasts, following ALP staining.

OARSI score. Cartilage damage was investigated by performing Safranin O/fast green staining (Fig. 2A). The results revealed no notable damage in the mild symptom group, mild damage in the moderate symptom group and severe damage in the severe symptom group. In the latter group, some of the subchondral bone was no longer covered by cartilage and the subchondral bone was completely exposed. The cartilage was then scored according to the severity of cartilage damage and in combination with the OARSI scoring system (OARSI score was equal to the cartilage degenerative tissue grading multiplied by the stage). Statistical analysis was then performed, with higher scores indicating more severe cartilage injury. The differences between the mild and moderate symptom groups, and 
A

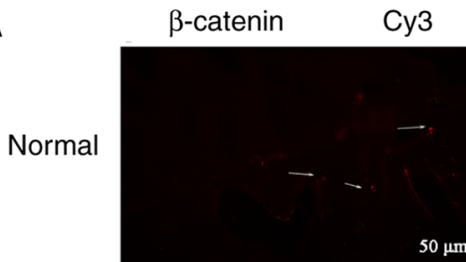

Mild

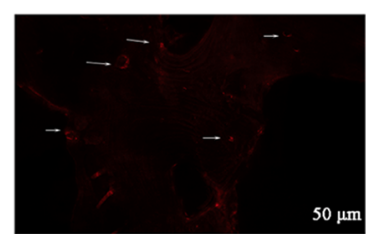

Moderate

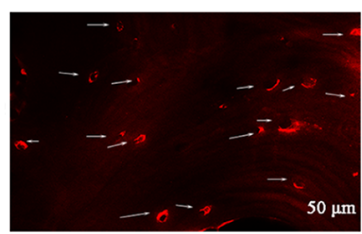

Severe

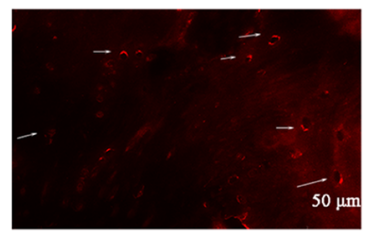

DAPI
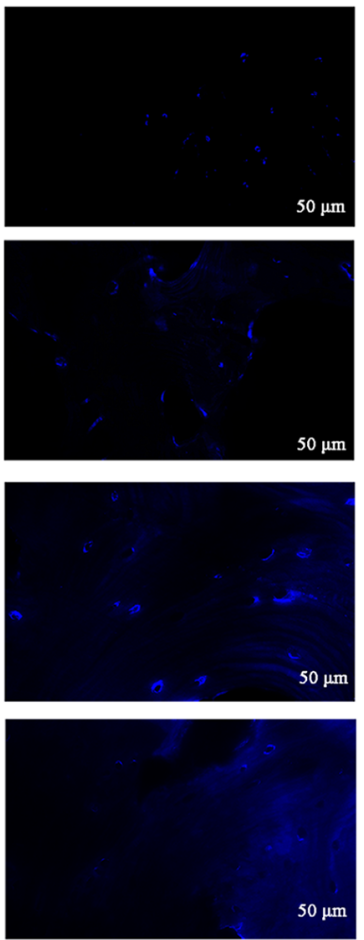
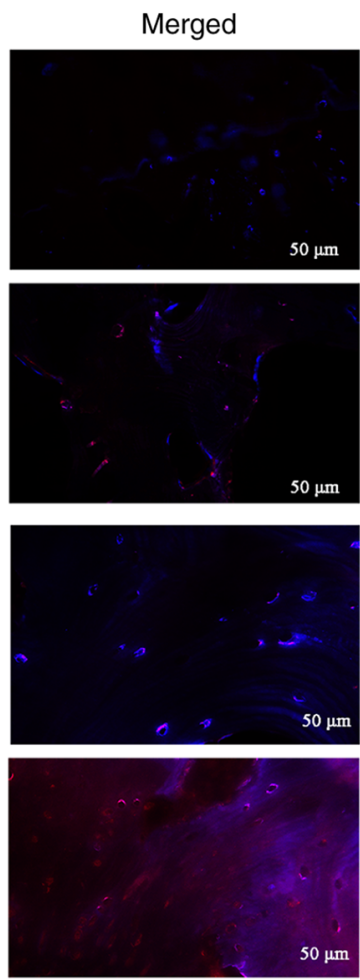

B

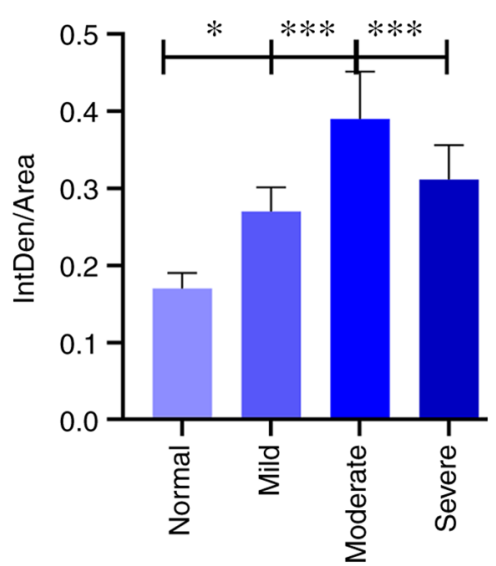

C $\beta$-catenin-IF Scatter chart

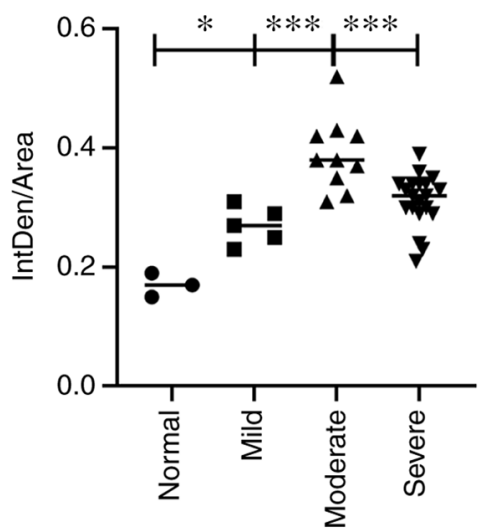

D IF- $\beta$-catenin

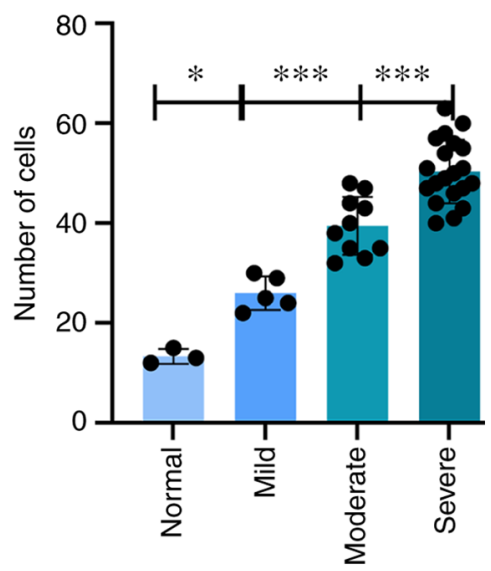

Figure 4. (A) Immunofluorescence staining ( $\beta$-catenin). Arrows indicate the number of positively stained cells. (B and C) Statistical analysis results. Statistical significant differences in b-catenin expression were revealed between normal and mild, mild and moderate, and moderate and severe OA in the $\beta$-catenin group ( $\mathrm{P}<0.05,{ }^{* * *} \mathrm{P}<0.001$ and ${ }^{* * * *} \mathrm{P}<0.001$, respectively). (D) Statistical analysis results of cell numbers. The number of cells was statistically significant between the normal and mild, mild and moderate, and moderate and severe symptom groups $\left({ }^{*} \mathrm{P}<0.05,{ }^{* * *} \mathrm{P}<0.001\right.$ and ${ }^{* * *} \mathrm{P}<0.001$, respectively). (B-D) Data were analyzed using one-way ANOVA. Mean 95\% confidence interval for average score: Normal $(n=3)$, mild $(n=5)$, moderate $(n=10)$ and severe $(n=20)$. OA, osteoarthritis

the moderate and severe symptom groups were statistically significant (both $\mathrm{P}<0.0001$; Fig. 2B).

$\beta$-catenin and DKK3 expression evaluation using immunohistochemistry. To observe the expression patterns of $\beta$-catenin and DKK3 in the osteoblasts in the subchondral bone, immunohistochemistry was performed, and the results were statistically analyzed (Fig. 3). For $\beta$-catenin (Fig. 3A), the differences in cell counts between the normal and mild groups, mild and moderate groups, and moderate and severe groups were statistically significant $(\mathrm{P}<0.05, \mathrm{P}<0.01$ and $\mathrm{P}<0.01$, respectively) (Fig. 3C). For DKK3 (Fig. 3B), there were statistically significant differences in cell numbers between the normal and mild, mild and moderate, and moderate and severe symptom groups $(\mathrm{P}<0.05, \mathrm{P}<0.01$ and $\mathrm{P}<0.05$, respectively) (Fig. 3E). In addition, the differences between the mild and moderate symptom groups were statistically significant according to the analysis of optical density values $(\mathrm{P}<0.001$ and $\mathrm{P}<0.01$, respectively; Fig. 3D and F).

Evaluation of $\beta$-catenin expression and DKK3 by immunofluorescence. To further observe $\beta$-catenin and DKK3 expression levels in osteoblasts from the subchondral bone, immunofluorescence staining was used. The results revealed the optical density changes in $\beta$-catenin and DKK3 expression in the subchondral osteoblasts. Immunofluorescence staining 
A
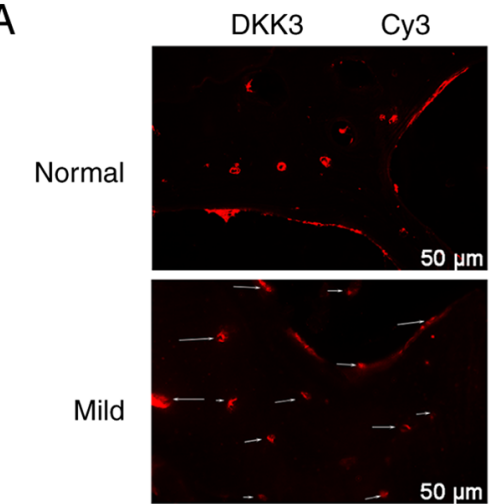

$50 \mu \mathrm{m}$

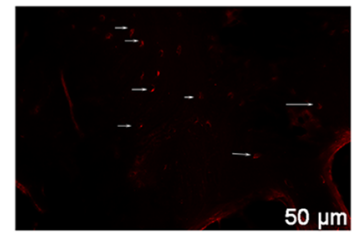

Moderate

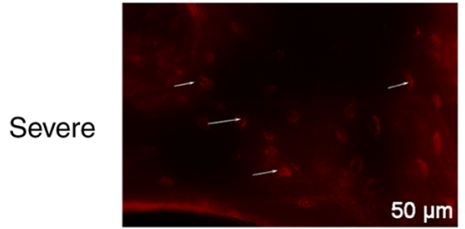

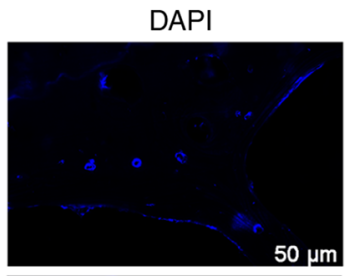
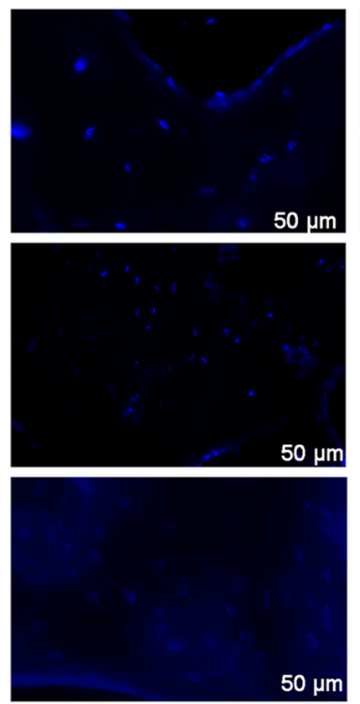

C

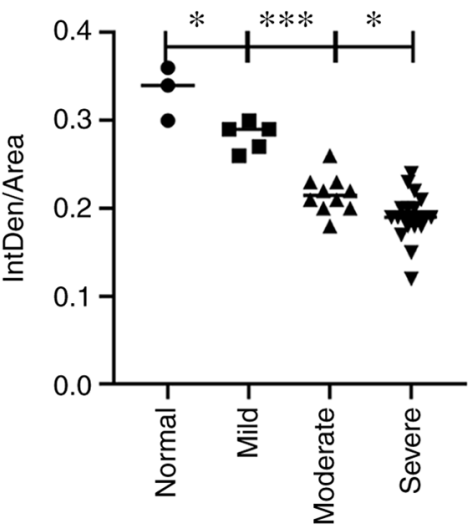

DKK3-IF Scatter chart
Merged
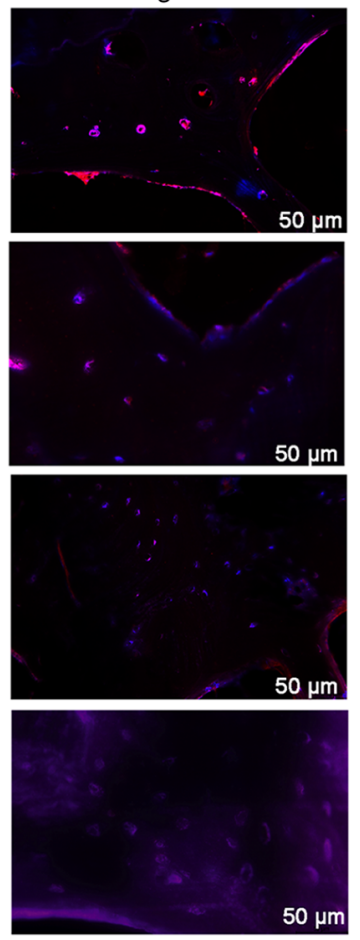
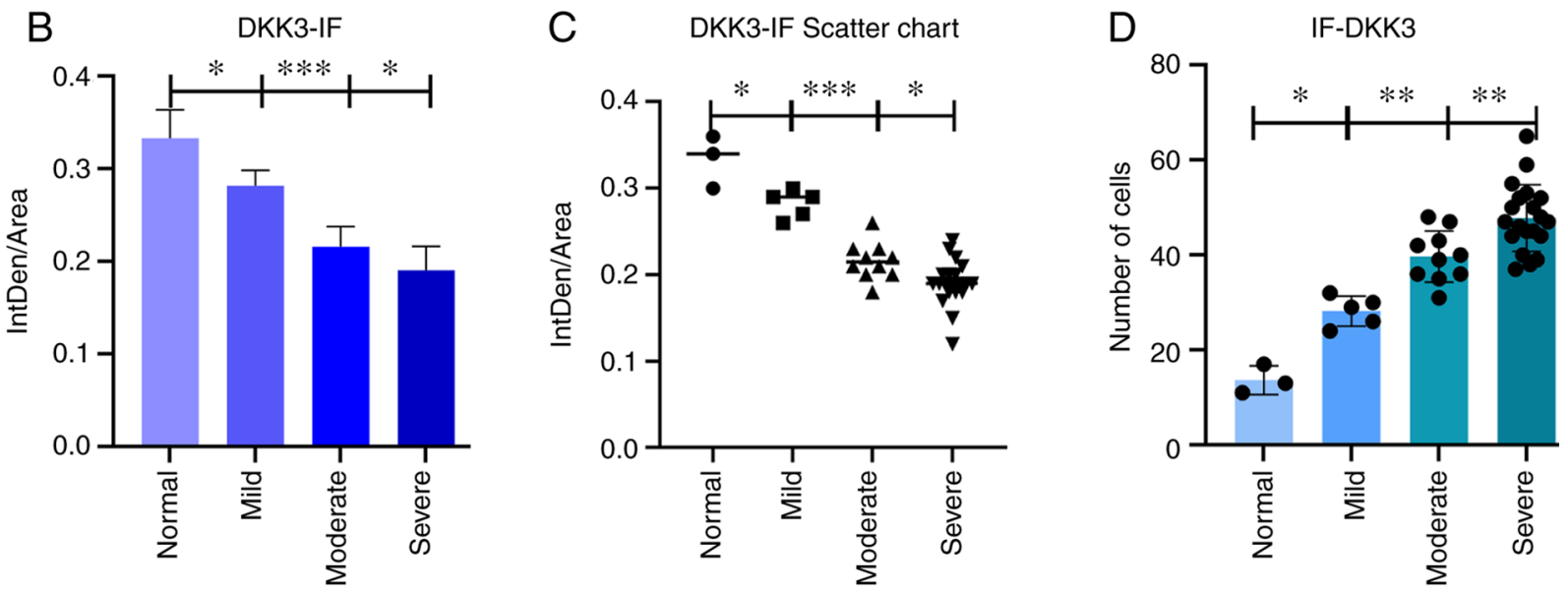

Figure 5. (A) Immunofluorescence staining for DKK3. Arrows indicate the number of positively stained cells. (B and C) Statistical analysis results. The difference in DKK3 expression between the normal and mild, mild and moderate, and moderate and severe groups was statistically significant $\left({ }^{*} \mathrm{P}<0.05\right.$, ${ }^{* * *} \mathrm{P}<0.001$ and ${ }^{*} \mathrm{P}<0.05$, respectively). (D) Cell count statistical analysis results. The differences in cell numbers was statistically significant between the normal and mild, mild and moderate, and moderate and severe symptom groups ( ${ }^{*} \mathrm{P}<0.05,{ }^{* *} \mathrm{P}<0.01$, and ${ }^{* *} \mathrm{P}<0.01$, respectively). (B-D) Data were analyzed using one-way ANOVA; mean 95\% confidence interval for average score: Normal $(n=3)$, mild $(n=5)$, moderate $(n=10)$ and severe $(n=20)$. DKK3, Dickkopf-related protein 3 .

was performed using $\mathrm{Cy} 3$, which is excited at $532 \mathrm{~nm}$ and emits orange light, then images of the samples were captured using a 40X fluorescence microscope. The results of $\beta$-catenin (Fig. 4A) and DKK3 expression (Fig. 5A) were observed and statistically analyzed (Figs. 4B-D, and 5B and C). The cell counts were statistically significantly different between the normal and mild, mild and moderate, and moderate and severe symptom groups. The differences in $\beta$-catenin and DKK3 expression were statistically significant between the mild and moderate symptom groups by analyzing the mean optical density $(\mathrm{P}<0.001$ and $\mathrm{P}<0.001$, respectively).

Changes in $\beta$-catenin and DKK3 protein and $m R N A$ expression. To detect and quantify $\beta$-catenin and DKK3 protein expression in the subchondral bone tissue, western blot analysis was performed. By analyzing the results (Fig. 6A-C) using statistical analysis, significant differences in the protein expression level of $\beta$-catenin were observed between the mild and moderate symptom groups, and between the moderate and severe symptom groups $(\mathrm{P}<0.001$ and $\mathrm{P}<0.01$, respectively). The $\beta$-catenin expression levels were more increased in the moderate symptom group. There were also statistically significant differences in the protein expression level of DKK3 between the mild and moderate symptom groups, and between the moderate and severe symptom groups $(\mathrm{P}<0.01$ and $\mathrm{P}<0.0001$, respectively). Increased DKK3 expression levels were observed in the normal and mild symptom groups, whereas decreased expression levels were observed in the 
A

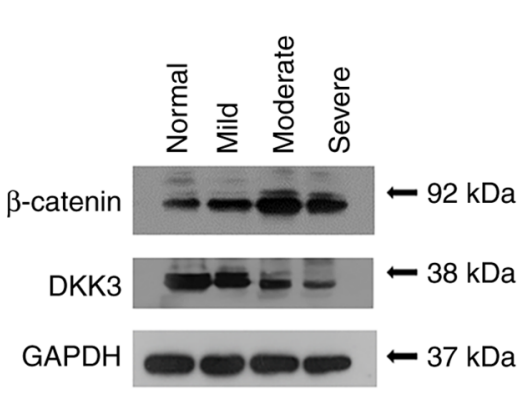

B

C

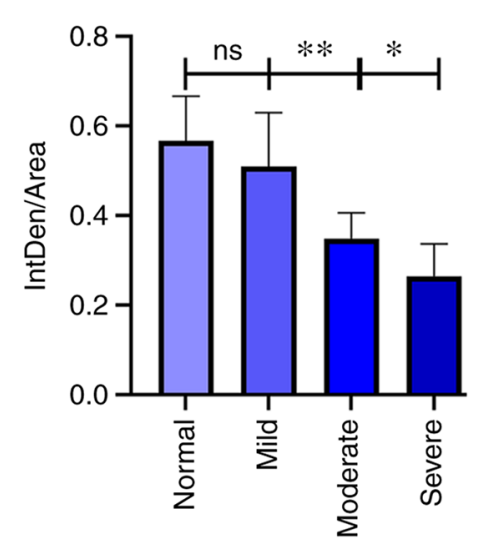

$\beta$-catenin-Wb

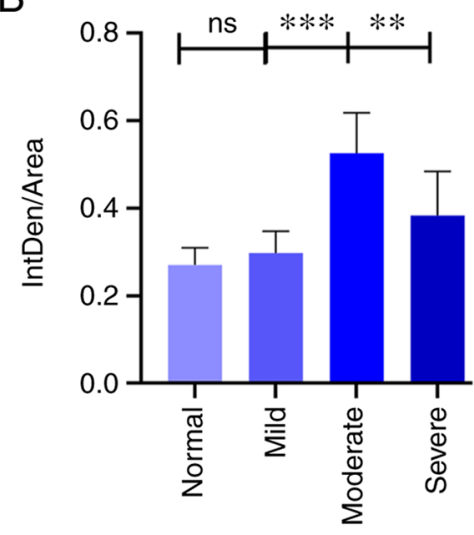

DKK3-Wb

E
$\beta$-catenin-Wb Scatter chart

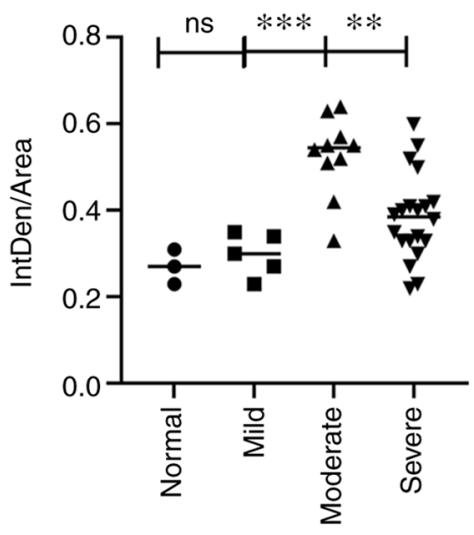

DKK3-Wb Scatter chart

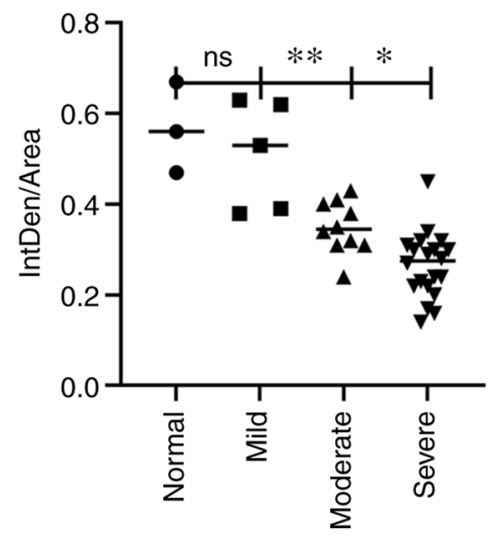

D

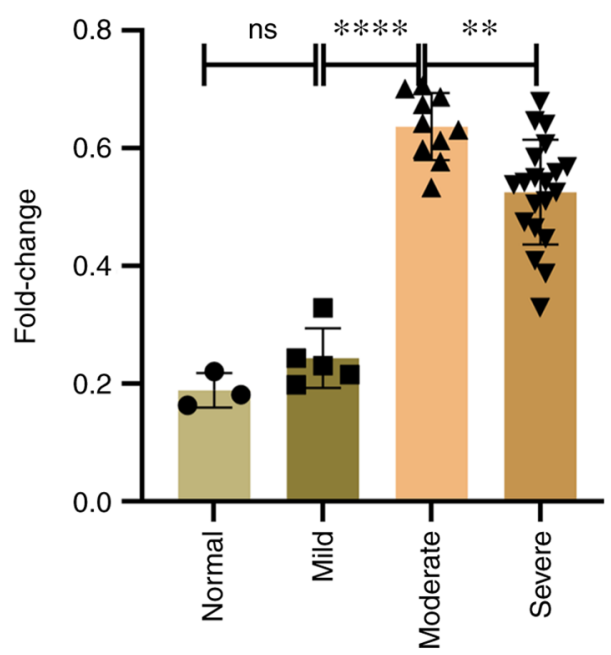

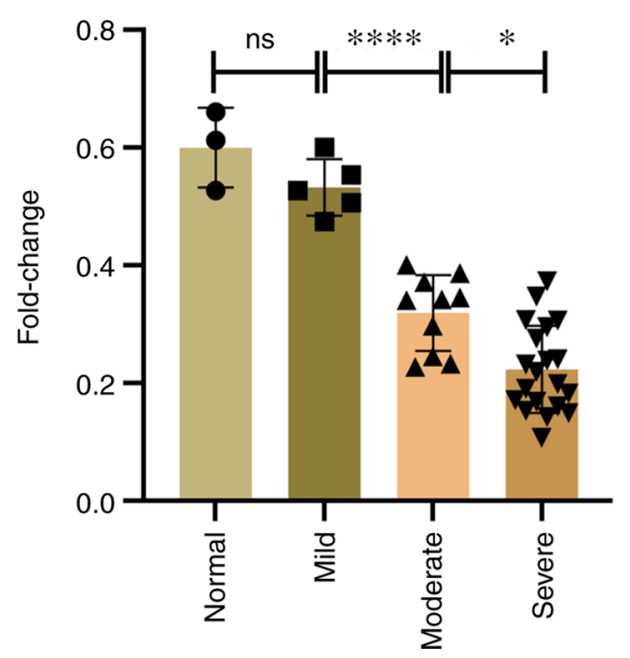

Figure 6. (A-C) The results of western blot analysis revealed that in the $\beta$-catenin group, there was a statistically significant difference between the mild and moderate symptom groups and between the moderate and severe symptom groups ${ }^{* * * *} \mathrm{P}<0.001$ and ${ }^{* *} \mathrm{P}<0.01$, respectively). In the DKK 3 group, there were statistically significant differences between the mild and moderate symptom groups, as well as between the moderate and severe symptom groups $\left({ }^{* *} \mathrm{P}<0.01\right.$ and ${ }^{*} \mathrm{P}<0.05$, respectively). (D and E) The results of RT-qPCR revealed significant differences between the mild and moderate groups as and between the moderate and severe symptom groups in the $\beta$-catenin group $\left({ }^{* * * * *} \mathrm{P}<0.0001\right.$, and ${ }^{* * *} \mathrm{P}<0.01$, respectively). In the DKK3 group, there was a statistically significant difference between the mild and moderate symptom groups and between the moderate and severe symptom groups ${ }^{* * * * *} \mathrm{P}<0.0001$ and ${ }^{*} \mathrm{P}<0.01$, respectively). (B-E) Data were analyzed using one-way ANOVA. $95 \%$ confidence interval for average score: Normal $(n=3)$, mild $(n=5)$, moderate $(n=10)$ and severe $(n=20)$. DKK3, Dickkopf-related protein 3; RT-qPCR, reverse transcription quantitative PCR; ns, not significant.

moderate and severe symptom groups, exhibiting a trend towards a decrease in expression levels with the severity of OA. Finally, in order to detect the gene expression level of $\beta$-catenin and DKK3 in the bone tissue from subchondral bone, RT-qPCR was performed. The results (Fig. 6D and E) demonstrated significant differences between the mild and 


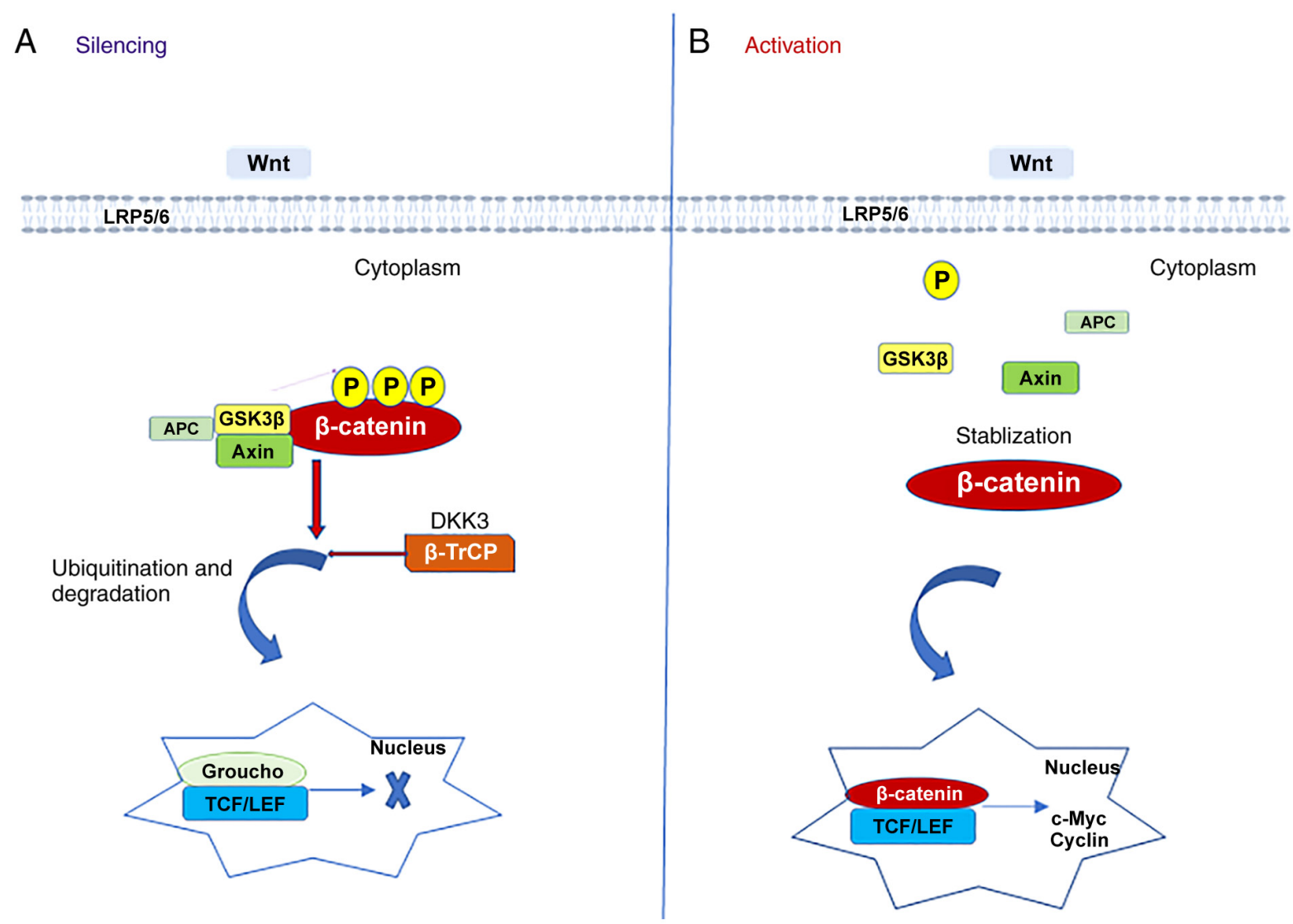

Figure 7. Overview of the Wnt signaling pathway. (A) Through the silencing the Wnt pathway, DKK3 competes with $\beta$-catenin in the cytoplasm and promotes its degradation, inhibiting the Wnt pathway and blocking nuclear transcription. (B) Activation of the Wnt pathway $\beta$-catenin aggregates in the cytoplasm. Stable $\beta$-catenin is then transferred to the nucleus and stimulates the transcription of Wnt target genes. DKK3, Dickkopf-related protein 3.

moderate symptom groups, and between the moderate and severe symptom groups concerning the $\beta$-catenin expression evaluation group $(\mathrm{P}<0.0001$ and $\mathrm{P}<0.01$, respectively). Concerning DKK3 mRNA expression, there was a statistically significant difference between the mild and moderate symptom groups and between the moderate and severe symptom groups $(\mathrm{P}<0.0001$ and $\mathrm{P}<0.01$ respectively), demonstrating an increased expression in the normal and mild symptom groups, and a decreased expression in the moderate and severe symptom groups, exhibiting a trending towards a decreased expression with the increasing severity of OA.

\section{Discussion}

Previous research has characterized early-stage OA with increased subchondral bone remodeling and late OA with decreased bone resorption and increased bone formation (5). Abnormal subchondral bone remodeling and osteophyte formation are hallmarks of OA progression (20).

Human $\beta$-catenin protein is an $88-\mathrm{kDa}$ cytoskeletal protein, 781-amino acid long, encoded by the catenin beta-1 gene (CTNNBI) and located on the 3p21 human chromosome (21). Numerous studies have shown that $\beta$-catenin is a major signaling molecule in the Wnt signaling pathway (22-24). Wnt signaling pathway activation depends on $\beta$-catenin expression levels in the cell (Fig. 7). When $\beta$-catenin levels are decreased, the Wnt pathway is not activated; however, when the levels are increased, the Wnt pathway is activated (25).
DKK3 is a member of the DKK family, among which DKK-1, -2 and -4 compete for binding to the Wnt protein low-density lipoprotein 5/6 receptor (26). DKK3 negatively regulates $\beta$-catenin and its downstream signaling pathways by reducing $\beta$-catenin aggregation in the cytoplasm (27). The graphs presented in Fig. 1 depict the changing patterns of $\beta$-catenin and DKK3 expression when the Wnt/ $\beta$-catenin pathway is inhibited and activated. In addition, DKK3 indirectly inhibited its role in the $\mathrm{Wnt} / \beta$-catenin pathway by reducing the nuclear transcription of $\beta$-catenin. Suwa et al (28) reported that various genes in the Wnt signaling pathway were expressed in the adrenal cortex. Based on the regional distribution pattern of DKK3 expression, it may be associated with the $\mathrm{Wnt} / \beta$-catenin signaling pathway, affecting regional differentiation or growth. However, it is unclear whether DKKs are associated with bone formation. Subsequently, Niehrs (29) reported that, in adults, DKKs were associated with bone formation in bone disease. Furthermore, Aslan et al (30) further demonstrated that DKK3 expression affected the osteogenesis process in vivo and in vitro, suggesting that it may exert an inhibitory effect on osteogenesis.

As the number of studies on the involvement of the Wnt signaling pathway in the developmental mechanisms of OA have increased, it has been reported that the activation of the classical Wnt/ $\beta$-catenin pathway accelerates the progression of OA (31). According to a previously published study by Funck-Brentano et al (32), it was revealed by using a mouse model of OA that the classic Wnt pathway was activated 
to promote subchondral bone formation osteophytes and inhibition of the $\mathrm{Wnt} / \beta$-catenin pathway signaling improved subchondral bone structure and biomechanical function, thereby alleviating the degeneration of articular cartilage. This may be attributed to active osteoblasts in OA altering bone microarchitecture through aberrant bone remodeling and reduced mineralization. However, this alteration notably reduced the biomechanical function of the subchondral bone, depriving it of its cushioning effect against mechanical loads and its protective function against cartilage, further aggravating cartilage damage and accelerating the development of OA.

In the present study, a total of 38 subchondral bone tissue specimens were collected from the tibial plateau in patients with severe OA. In addition, intraoperative anatomical tissues and postoperative tibial plateau osteotomy specimens were also collected, demonstrating severe cartilage damage in the femoral condyle and tibial plateau, with some areas of cartilage lost and replaced by sclerotic subchondral bone. H\&E and ALP staining of the subchondral bone tissue revealed that the total number of osteocytes and osteoblasts in the subchondral bone increased along with the degree of $\mathrm{OA}$, including various osteoblasts (pre-osteoblasts, osteoclasts and bone lining cells). Neve et al (4) reported that the abnormal differentiation and increased activity of osteoblasts were observed in OA. In addition, dysfunctional osteoblasts produce in turn numerous transcription factors, growth factors and other proteins involved in the pathogenesis of OA (33), further promoting the development of OA.

It has been found that osteoblast differentiation requires the classical Wnt/ $\beta$-catenin signaling pathway (14). It has been reported that some osteoblasts contain ALP, which can be used as a biochemical marker to assess bone formation (34); however, mechanical loading increases ALP activity (35). Thus, in the present study, it was demonstrated using ALP staining (AZO method) that the number of ALP-positive cells in the subchondral bone specimens, to be increased during the progression of OA from mild to moderate; in addition, in severe OA, there was no significant increase in active osteoblasts compared with that in moderate OA.

Cartilage changes may be visualized using Safranin O/fast green staining, revealing that the hyaline cartilage cracks, thins and eventually disappears, as the degree of OA increases. It has been reported that articular cartilage is very sensitive to mechanical loading, subchondral bone provides cartilage support and cartilage degeneration is a sign of OA aggravation (36). The role of the subchondral bone in the development of OA remains under investigation; however, there is evidence to indicate that changes in the subchondral bone are accompanied by cartilage degeneration (37).

Another study revealed that metabolic modulators produced by subchondral osteoblasts directly cause chondrocyte degeneration by affecting the microstructure between the bone and cartilage, while abnormal expression of osteoblasts can indirectly expose cartilage to higher stress (38). Thus, this direct and indirect mode of action of subchondral bone and cartilage accelerates cartilage degeneration and further aggravates the progression of OA. Fell et al (39) reported that the association between the morphology of the subchondral bone tissue and the viscoelasticity, and thickness of cartilage may contribute to the development and progression of
OA by altering the ability of cartilage to dissipate energy. Furthermore, Smieszek et al (40) demonstrated that osteoblasts are inhibited by high expression of microRNA-21 in OA, through the reduction of subchondral bone mineralization. Therefore, altering and alleviating the abnormal remodeling of subchondral bone may mitigate further cartilage damage and delay the progression of OA.

Due to this supportive role of subchondral bone on cartilage, the question on whether DKK3 could reduce subchondral bone sclerosis via $\beta$-catenin inhibition of the Wnt pathway, preventing further damage to cartilage due to subchondral bone sclerosis and delaying the progression of OA was raised. Among the numerous regulators of the classical Wnt pathway, $\beta$-catenin is a key factor, while DKK3 is a downstream inhibitor of the Wnt pathway. In malignant tumor cells, DKK3 was associated with the induction of apoptosis and inhibition of invasion by regulating $\beta$-catenin signaling, and c-Jun $\mathrm{N}$-terminal kinase-dependent cellular pathways, as reported by Lee et al (17). It has been reported that $\beta$-catenin may be a central molecule mediating osteoblast viability and differentiation (41). In addition, DKK3 inhibited the Wnt signaling pathway by hydrolyzing $\beta$-catenin/APC/GSK3 $\beta$, thereby inhibiting the entry of $\beta$-catenin into the nucleus for transcription (17). Therefore, in OA, DKK3 degrades $\beta$-catenin via ubiquitination, thereby degrading the downstream signaling pathway factors of $\beta$-catenin and inhibiting nuclear transcription of the Wnt pathway (15), delaying abnormal remodeling of subchondral bone.

According to Huang et al (42) the regulation of the Wnt signaling pathway reduced the risk of OA. Charlier et al (43) reported that the DKK3 regulation of the $\mathrm{Wnt} / \beta$-catenin pathway increased the dedifferentiation of OA chondrocytes in the human hip and promoted the remission of OA. Thus, maintaining normal Wnt/ $\beta$-catenin signaling is critical for orthogenic bone tissue differentiation and in $\mathrm{OA}$, once this pathway is activated, it can accelerate OA progression.

In conclusion, the results of the present study demonstrated that with the increase in the pathological changes of $\mathrm{OA}$ in the knee, the number of osteoblasts may also gradually increase; however, the number of active osteoblasts was highest in moderate OA and slightly more increased in severe OA. The aforementioned association analysis revealed that DKK3 expression was high in the normal and mild groups, decreased in the moderate and severe groups, while $\beta$-catenin expression increased significantly in the moderate OA group after activation of the Wnt/ $\beta$-catenin pathway. In subchondral bone tissue, DKK3 may play an opposite role to $\beta$-catenin by inhibiting the $\mathrm{Wnt} / \beta$-catenin signaling pathway in osteoblasts, reducing subchondral bone sclerosis, while reducing cartilage damage and delaying OA progression. In future studies, the authors aim to verify whether DKK3 can inhibit bone formation in vitro. Furthermore, it was unexpectedly found that, in the late stages of OA, some osteoblasts can still be activated and play a role in abnormal bone remodeling, leading to increased subchondral bone sclerosis and worsening of pain.

\section{Acknowledgements}

Not applicable. 


\section{Funding}

The present study was supported by the National Natural Science Foundation of China (grant no. 8186090221).

\section{Availability of data and materials}

The datasets used and/or analyzed during the current study are available from the corresponding author on reasonable request.

\section{Authors' contributions}

$\mathrm{XL}$ and QJ contributed to the conception and design of the present study. XL, WJ and XY performed the experiments. XL performed the statistical analysis and contributed to the manuscript preparation. QJ and XL confirm the authenticity of all the raw data. QJ contributed to funding acquisition. All authors have read and approved the final version of the manuscript.

\section{Ethics approval and consent to participate}

The present study was approved by the Ethics Committee of the General Hospital of Ningxia Medical University (approval no. KYLL-2020-20; approval date, January 14, 2021), and informed consent was obtained from all the enrolled patients.

\section{Patient consent for publication}

The patient whose images are shown in Fig. 1 consents to the publication of these images.

\section{Competing interests}

The authors declare that they have no competing interests.

\section{References}

1. Qin HJ, Xu T, Wu HT, Yao ZL, Hou YL, Xie YH, Su JW, Cheng CY, Yang KF, Zhang XR, et al: SDF-1/CXCR4 axis coordinates crosstalk between subchondral bone and articular cartilage in osteoarthritis pathogenesis. Bone 125: 140-150, 2019.

2. Anderson-MacKenzie JM, Quasnichka HL, Starr RL, Lewis EJ, Billingham ME and Bailey AJ: Fundamental subchondral bone changes in spontaneous knee osteoarthritis. Int J Biochem Cell Biol 37: 224-236, 2005.

3. Omoumi P, Babel H, Jolles BM and Favre J: Relationships between cartilage thickness and subchondral bone mineral density in non-osteoarthritic and severely osteoarthritic knees: In vivo concomitant $3 \mathrm{D}$ analysis using $\mathrm{CT}$ arthrography. Osteoarthritis Cartilage 27: 621-629, 2019.

4. Neve A, Corrado A and Cantatore FP: Osteoblast physiology in normal and pathological conditions. Cell Tissue Res 343: 289-302, 2010

5. Maruotti N, Corrado A and Cantatore FP: Osteoblast role in osteoarthritis pathogenesis. J Cell Physiol 232: 2957-2963, 2017.

6. Di Pompo G, Errani C, Gillies R, Mercatali L, Ibrahim T, Tamanti J, Baldini N and Avnet S: Acid-induced inflammatory cytokines in osteoblasts: A guided path to osteolysis in bone metastasis. Front Cell Dev Biol 9: 678532, 2021.

7. Wang Y, Fan X, Xing L and Tian F: Wnt signaling: A promising target for osteoarthritis therapy. Cell Commun Signal 17: 97, 2019.

8. Lories RJ and Monteagudo S: Review article: Is Wnt signaling an attractive target for the treatment of osteoarthritis? Rheumatol Ther 7: 259-270, 2020.
9. Cherifi C, Monteagudo S and Lories RJ: Promising targets for therapy of osteoarthritis: A review on the Wnt and TGF- $\beta$ signalling pathways. Ther Adv Musculoskel 13: 1759720X211006959, 2021.

10. Chen H, Tan XN, Hu S, Liu RQ, Peng LH, Li YM and Wu P: Molecular mechanisms of chondrocyte proliferation and differentiation. Front Cell Dev Biol 9: 664168, 2021.

11. Staines KA, Macrae VE and Farquharson C: Cartilage development and degeneration: A Wnt Wnt situation. Cell Biochem Funct 30: 633-642, 2012.

12. Hill TP, Später D, Taketo MM, Birchmeier W and Hartmann C: Canonical Wnt/beta-catenin signaling prevents osteoblasts from differentiating into chondrocytes. Dev Cell 8: 727-738, 2005.

13. Zhu M, Tang D, Wu Q, Hao S, Chen M, Xie C, Rosier RN, O'Keefe RJ, Zuscik M and Chen D: Activation of beta-catenin signaling in articular chondrocytes leads to osteoarthritis-like phenotype in adult beta-catenin conditional activation mice. J Bone Miner Res 24: 12-21, 2009.

14. Fjeld K, Kettunen P, Furmanek T, Kvinnsland IH and Luukko K: Dynamic expression of Wnt signaling-related Dickkopf 1, -2, and -3 mRNAs in the developing mouse tooth. Dev Dyn 233: $161-166,2005$.

15. Chen J, Yang C, Yang Y, Liang Q, Xie K, Liu J and Tang Y: Targeting DKK1 prevents development of alcohol-induced osteonecrosis of the femoral head in rats. Am J Transl Res 13: 2320-2330, 2021.

16. Baetta R and Banfi C: Dkk (Dickkopf) proteins. Arterioscler Thromb Vasc Biol 39: 1330-1342, 2019.

17. Lee EJ, Nguyen QTT and Lee M: Dickkopf-3 in human malignant tumours: A clinical viewpoint. Anticancer Res 40: 5969-5979, 2020.

18. Pritzker KP, Gay S, Jimenez SA, Ostergaard K, Pelletier JP, Revell PA, Salter D and van den Berg WB: Osteoarthritis cartilage histopathology: Grading and staging. Osteoarthritis Cartilage 14: 13-29, 2006.

19. Livak KJ and Schmittgen TD: Analysis of relative gene expression data using real-time quantitative PCR and the 2(-Delta Delta C(T)) method. Methods 25: 402-408, 2001.

20. Zhang L and Wen C: Osteocyte dysfunction in joint homeostasis and osteoarthritis. Int J Mol Sci 22: 6522, 2021.

21. Tian J, Gao SG, Li YS, Cheng C, Deng ZH, Luo W and Zhang FJ: The $\beta$-catenin/TCF-4 pathway regulates the expression of OPN in human osteoarthritic chondrocytes. J Orthop Surg Res 15: 344, 2020.

22. Li W, Xiong Y, Chen $\mathrm{W}$ and $\mathrm{Wu} \mathrm{L}$ : Wnt $/ \beta$-catenin signaling may induce senescence of chondrocytes in osteoarthritis. Exp Ther Med 20: 2631-2638, 2020.

23. Xuan F, Yano F, Mori D, Chijimatsu R, Maenohara Y, Nakamoto H, Mori Y, Makii Y, Oichi T, Taketo MM, et al: Wnt/ $\beta$-catenin signaling contributes to articular cartilage homeostasis through lubricin induction in the superficial zone. Arthritis Res Ther 21: 247, 2019.

24. Yu H, Liu Y, Yang X, He J, Zhang F, Zhong Q and Guo X: Strontium ranelate promotes chondrogenesis through inhibition of the Wnt/ $\beta$-catenin pathway. Stem Cell Res Ther 12: 296, 2021.

25. Le NH, Franken P and Fodde R: Tumour-stroma interactions in colorectal cancer: Converging on beta-catenin activation and cancer stemness. Brit J Cancer 98: 1886-1893, 2008.

26. Ren C, Gu X, Li H, Lei S, Wang Z, Wang J, Yin P, Zhang C, Wang $\mathrm{F}$ and Liu C: The role of DKK1 in Alzheimer's disease: A potential intervention point of brain damage prevention? Pharmacol Res 144: 331-335, 2019.

27. Uribe D, Cardona A, Esposti DD, Cros MP, Cuenin C, Herceg Z, Camargo M and Cortés-Mancera FM: Antiproliferative effects of epigenetic modifier drugs through E-cadherin up-regulation in liver cancer cell lines. Ann Hepatol 17: 444-460, 2018.

28. Suwa T, Chen M, Hawks CL and Hornsby PJ: Zonal expression of dickkopf-3 and components of the Wnt signalling pathways in the human adrenal cortex. J Endocrinol 178: 149-158, 2003.

29. Niehrs C: Function and biological roles of the Dickkopf family of Wnt modulators. Oncogene 25: 7469-7481, 2006.

30. Aslan H, Ravid-Amir O, Clancy BM, Rezvankhah S, Pittman D, Pelled G, Turgeman G, Zilberman Y, Gazit Z, Hoffmann A, et al: Advanced molecular profiling in vivo detects novel function of dickkopf-3 in the regulation of bone formation. J Bone Miner Res 21: 1935-1945, 2006.

31. De Palma A and Nalesso G: WNT signalling in osteoarthritis and its pharmacological targeting. Handb Exp Pharmacol 269: 337-356, 2021. 
32. Funck-Brentano T, Bouaziz W, Marty C, Geoffroy V, Hay E and Cohen-Solal M: Dkk-1-mediated inhibition of Wnt signaling in bone ameliorates osteoarthritis in mice. Arthritis Rheumatol 66 : 3028-3039, 2014.

33. Jiang A, Xu P, Sun S, Zhao Z, Tan Q, Li W, Song C and Leng H: Cellular alterations and crosstalk in the osteochondral joint in osteoarthritis and promising therapeutic strategies. Connect Tissue Res 62: 709-719, 2021.

34. Liao F, Hu X and Chen R: The effects of omarigliptin on promoting osteoblastic differentiation. Bioengineered 12 : 11837-11846, 2021.

35. Miyamoto S, Yoshikawa $\mathrm{H}$ and Nakata K: Axial mechanical loading to ex vivo mouse long bone regulates endochondral ossification and endosteal mineralization through activation of the BMP-Smad pathway during postnatal growth. Bone Rep 15: 101088, 2021.

36. Vincent TL and Wann AKT: Mechanoadaptation: Articular cartilage through thick and thin. J Physiol 597: 1271-1281, 2019.

37. Bhatla JL, Kroker A, Manske SL, Emery CA and Boyd SK: Differences in subchondral bone plate and cartilage thickness between women with anterior cruciate ligament reconstructions and uninjured controls. Osteoarthritis Cartilage 26: 929-939, 2018.

38. Hu W, Chen Y, Dou C and Dong S: Microenvironment in subchondral bone: Predominant regulator for the treatment of osteoarthritis. Ann Rheum Dis 80: 413-422, 2020.

39. Fell NLA, Lawless BM, Cox SC, Cooke ME, Eisenstein NM, Shepherd DET and Espino DM: The role of subchondral bone, and its histomorphology, on the dynamic viscoelasticity of cartilage, bone and osteochondral cores. Osteoarthritis Cartilage 27: 535-543, 2019.
40. Smieszek A, Marcinkowska K, Pielok A, Sikora M, Valihrach L and Marycz K: The role of miR-21 in osteoblasts-osteoclasts coupling in vitro. Cells 9: 479, 2020.

41. Chu Y, Gao Y, Yang Y, Liu Y, Guo N, Wang L, Huang W, Wu L, Sun $\mathrm{D}$ and $\mathrm{Gu} \mathrm{W}$ : $\beta$-Catenin mediates fluoride-induced aberrant osteoblasts activity and osteogenesis. Environ Pollut 265: $114734,2020$.

42. Huang Y, Jiang L, Yang $\mathrm{H}, \mathrm{Wu} \mathrm{L}, \mathrm{Xu} \mathrm{N}$, Zhou X and Li J: Variations of Wnt $/ \beta$-catenin pathway-related genes in susceptibility to knee osteoarthritis: A three-centre case-control study. J Cell Mol Med 23: 8246-8257, 2019.

43. Charlier E, Malaise O, Deroyer C, Zeddou M, Neuville S, Cobraiville G, Gillet P, Kurth W, de Seny D, Relic B and Malaise MG: Dickkopf 3 (DKK3) is increased along human hip OA chondrocytes dedifferentiation and can modulate Wnt/B-catenin and TGF $\beta$ Alk1/Smad1/5 signaling pathways, as well as leptin production. Osteoarthritis Cartilage 24 (Suppl 1): S182, 2016. International (CC BY-NC-ND 4.0) License. 